\title{
Spinal cord lesion after penicillin gluteal injection
}

\author{
${ }^{1} \mathrm{~L}$ Tesio MD, ${ }^{1} \mathrm{~L}$ Bassi MD, ${ }^{2} \mathrm{~L}$ Strada MD \\ ${ }^{1}$ Servizio di Medicina Fisica e Riabilitazione, Divisione di Ortopedia e Traumatologia, \\ Istituto Scientifico Ospedale San Raffaele, Via Olgettina 60, 20132 Milano, Italy; \\ ${ }^{2}$ Divisione di Neuroradiologia, Istituto Neurologico 'C. Besta', Via Celoria, 20133 \\ Milano, Italy.
}

Penicillin gluteal injection may cause sudden and irreversible paraplegia. In the literature 6 cases have been reported, and spinal infarction was conjectured, the mechanism being obscure. The actual incidence of such a complication is not known.

We observed 2 more cases, 6 and 16 years respectively following such an injection. The clinical features suggested that spinal infarction had occurred, and an MRI study in the most severely impaired patient showed marked spinal atrophy, giving further support to the spinal infarction hypothesis. These findings and the pathophysiological considerations suggest that the mechanism might be the accidental injection into the superior gluteal artery, causing its distal spasm and the upstream ascent of the penicillin with ensuing embolic and/or spastic occlusion of the arterior spinal artery.

Key words: penicillin; paraplegia; spinal cord atrophy.

\section{Introduction}

Intragluteal injection of penicillin may be followed by sudden transverse myelitis and irreversible paraplegia: 6 cases have been previously described in the literature. ${ }^{1-5}$ The actual incidence of such a rare complication is not known, and the underlying mechanisms are still debatable. We described 2 further cases, observed 6-16 years after the accident, and in whom spinal MRI was performed.

\section{Case 1}

A woman aged 31 years. From the age of 20 she was treated with benzatin-penicillin $G$ (Diaminocillina $^{\mathrm{R}}$ ) for chronic endocarditis. When aged 25 , one minute after the injection of the antibiotic into the left buttock she developed paraplegia, incontinence, and a sensory loss up to midthoracic level. Ecchymosis extended from the injection site up to the left lumbar paraspinal area.

Sensation and continence recovered fully in a month. Motor power partially recovered. Six years after the accident she was still wheelchair bound, with flaccid paralysis of the pelvic and lower limb muscles (inner- vation distal to T11). Motor power was graded $2-3 / 5$ in the glutei, the right iliopsoas, the quadriceps and the peronei. A $0.5 \mathrm{~T}$ MRI was performed, with sagittal and coronal FFE $90^{\circ}$ sequences and sagittal multiple echoes. It showed marked atrophy of the thoraco-lumbar region of the spinal cord, without signal abnormalities (Fig 1).

\section{Case 2}

A man aged 25 years. From the age of 3 , following rheumatic fever and arthritis, he was treated with benzatin-benzil-penicillin $\left(\right.$ Wycillina $^{\mathrm{R}}$ ).

At the age of 9, a few seconds after injection of that antibiotic into the left buttock, he complained of acute pain down the left lower limb for some 3 minutes, and this limb became paralysed. Thereafter, for about 30 minutes he exhibited flexor spasms of both lower limbs, followed by paraplegia, incontinence, and anaesthesia up to the midthoracic level. Sensation recovered fully in a month; full control of micturiton required 13 years. Gait with the aid of orthopaedic shoes was possible in a year, but muscle strength recovered only par- 


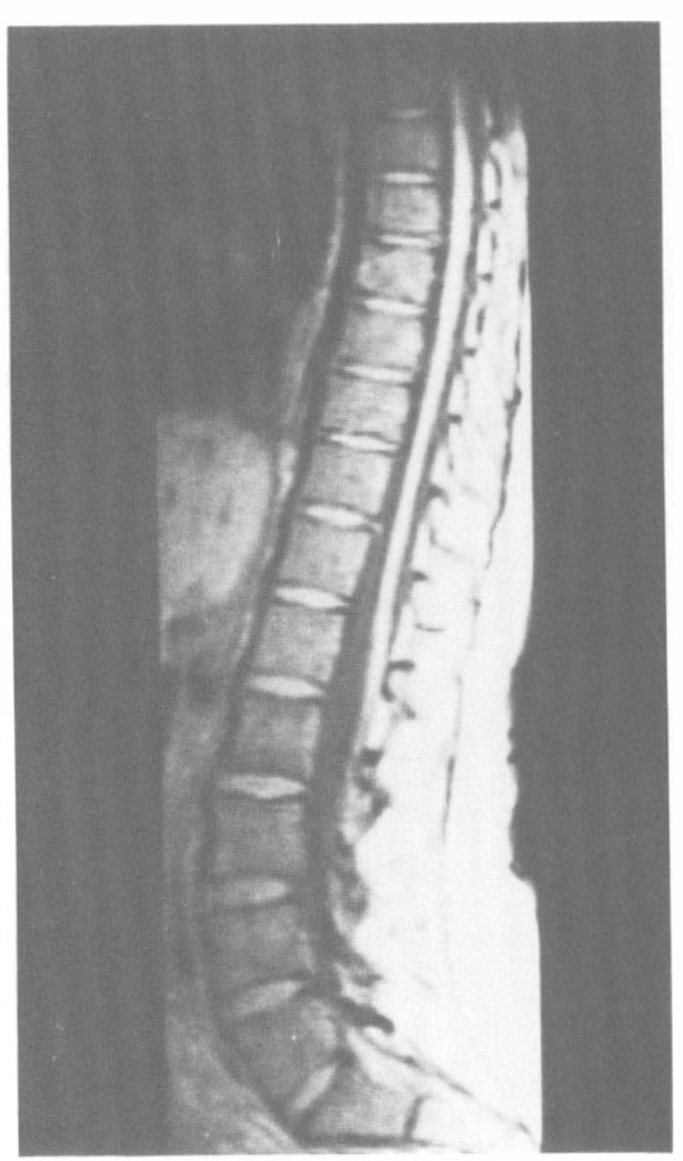

Figure 1 Spinal MR $-0.5 \mathrm{~T}, \mathrm{FFE} 90^{\circ}$, TR 410 TE $17-$ in a 31 year old women, performed 6 years after acute paraplegia following penicillin gluteal injection. Marked atrophy of the thoraco-lumbar cord is evidenced.

tially. A severe progressive right convex thoraco-lumbar scoliosis developed. Sixteen years after the accident the scoliosis had reached $70^{\circ}$, both lower limbs were hypotrophic, and the residual motor power of the gluteal, thigh and leg muscles (innervation distal to L2) ranged between 2 and $4 / 5$, except for the left tibialis anterior which was paralysed. Because of muscle contractures and bony deformities, he required operations on the lower limbs. MRI examination of the spinal cord (the same technique as in Case 1) appeared to be normal.

\section{Discussion}

To our knowledge only 6 similar cases have been reported in the literature ( 4 adults and 2 children of 15 months and $4 \frac{1}{2}$ years, respectively). In all of them an intragluteal injection of penicillin was suddenly followed by transverse myelitis, ${ }^{1,2,6-8}$ with neither signs nor symptoms of anaphylaxis. One patient died after 18 days, ${ }^{6}$ and the autopsy revealed selective haemorrhagic necrosis of the anterior horns of the lumbosacral cord. The 5 survivors remained paraplegic. In 2 of them ${ }^{2}$ myelography was performed, showing normal appearances.

All of the above cited authors agreed with the hypothesis of infarction of the thoracolumbar cord. The conjectured mechanism was an accidental injection into the superior gluteal artery, propelling the drug retrogradally through the internal iliac artery and its lumbar branch, or through the lumbar aorta, up to the origin of the anterior spinal artery of Adamkiewicz. It still remains a matter of debate whether ischaemia was finally due to localised arterial occlusion or to extensive arterial spasm.

Together with previous autopsy findings, our 2 cases appear to support the former hypothesis. The neurological late sequelae of flaccid paralysis or muscle weakness, with no sensory, sphincterial or pyramidal signs were consistent with the anterior spinal cord syndrome. In fact, typical results of the occlusion of the Adamkiewicz's artery are isolated muscle deficits, due to the selective necrosis of the grey matter in the anterior horns, which are particularly sensitive to ischaemia. ${ }^{6}$

In addition, in the first of our 2 patients, who was indeed more severely neurologically impaired, the MRI showed evidence of spinal cord damage, with marked atrophy of the thoraco-lumbar cord, consistent with late effects of a vascular lesion (Fig 1). In the second, less neurologically impaired patient, the MRI findings appeared to be normal, but neurological examination suggested a more scattered and partial damage to the anterior horns in the cord.

If an arterial retrograde embolism is a tenable mechanism, then embolic accidents after penicillin gluteal injection might be expected to occur also in the lower limbs, 
and also after injection of other drugs. Indeed some cases have been reported in which either penicillin or bismuth injections were followed by gluteal or lower limb gangrene, ${ }^{4,5}$ and this raises the question whether the active drugs themselves or other components of the injected solutions were responsible for the complication.

The mechanism seems to be analogous to that proposed by Cohen for upper limb gangrene following accidental injection of thiopentone ${ }^{7}$ or quinine ${ }^{8}$ into brachial arteries: he calculated an incidence of about 1 in 55,000. He also warned that 'this accident can happen to anyone', since intraarterial injection can be painless, and often it 'is not followed, as is commonly imagined, by a gush of blood blowing off the syringe piston'. ${ }^{3}$ In the same work, he also demonstrated on a simple hydraulic model that fluid can be easily pushed in a retrograde manner against the arterial pressure for as much as $12 \mathrm{~cm}$, the ascent being higher, and of longer duration, the lower is the arterial flow.

Once extrapolated to the case of gluteal injection, Cohen's model might support the hypothesis of a retrograde spinal embolism either in children ${ }^{1,5}$ or in adults, ${ }^{4}$ albeit in the latter a concurrent distal spasm of the gluteal artery might be claimed as a relevant factor.

\section{References}

1 Atkinson JP (1969) Transverse myelopathy secondary to injection of penicillin. J. Pediatr 75: 867-872.

2 Barone A, Dell'Aria V (1972) Sindromi di lesione midollare trasversa da iniezione di penicillina. Acta Neurol (Napoli) 27: 379-385.

3 Pasco GF, Zanetti R (1976) Considerazioni su di un caso di lesione midollare trasversa da iniezione intraglutea di penicillina ritardo. Riv Neurol 22(5): 59-67.

4 Perulli L, Fontanari D (1978) Mielopatie da iniezione di penicillina ritardo. Presentazione di un caso e revisione della letteratura. Riv Neurobiol 24(7): 343-359.

5 Shaw EB (1966) Transverse myelitis from injection of penicillin. Am J Dis Child 111: 548-551.

6 Dodson EW, Landau WM (1973) Motor neuron loss due to aortic clamping in repair of coarctation. Neurology 23(5): 539-542.

7 Cohen M (1948) Accidental intra-arterial injection of drugs. Lancet 2: 361-371.

8 Cohen M (1948) Accidental intra-arterial injection of drugs. Dangers of therapeutic or diagnostic intra-arterial injections. Lancet 2: 409-416. 